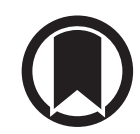

CrossMark

\section{Passive smoking in relation to lung cancer incidence and histologic types in Norwegian adults: the HUNT study}

\author{
To the Editor:
}

Passive smoking has been proposed as a risk factor for lung cancer. The increased risks for lung cancer overall and different histologic types in relation to passive smoking were demonstrated in two meta-analytical studies $[1,2]$, in which the included studies were mostly case-control designs, which are subject to recall bias. To date, there have been a limited number of prospective studies on passive smoking in relation to lung cancer incidence, most of which included only never-smoking women [3-5]. There is also a lack of longitudinal investigations concerning the influence of passive smoking on different histologic types of lung cancer. We aimed to evaluate the influences of passive smoking during childhood and adulthood on the development of lung cancer and histologic types in a long-term follow-up cohort, and to assess possible sex-related differences in the association.

We used the Nord-Trøndelag Health Survey 2 (HUNT2), conducted in 1995-1997, including 65229 participants aged 20 years or older. Exposure and other baseline variables were collected by referring to the questionnaires or clinical examinations conducted in HUNT2. Participants were grouped into four passive smoking categories (never, in childhood only, in adulthood only, in both periods) based on two questions - i.e. "Did any of the adults smoke indoors where you grew up?", and "After you were 20 years old, do you live or have you lived with daily smoker(s)?" Using the unique 11-digit personal identification number, the HUNT2 population data were linked with data from the Cancer Registry of Norway. The ICD-10 (Tenth Revision of the International Statistical Classification of Diseases and Related Health Problems) topography codes used for registration of lung cancer were C33-C34 [6]. Histologic types were classified according to the International Classification of Diseases of Oncology (ICD-O) [7]. We excluded subjects with missing information on active or passive smoking $(\mathrm{n}=2300)$, subjects with ever cancer reported in HUNT2 ( $\mathrm{n}=6144)$, or lung cancer cases diagnosed before the participation date in HUNT2 $(\mathrm{n}=13)$, leaving 56772 subjects remaining in the current analyses.

Competing-risks regression models were applied to estimate the subdistribution hazard ratios (SHRs) with 95\% confidence intervals for lung cancer overall and histologic types in association with passive smoking [8]. Death due to other reasons and the other lung cancer subtypes were considered as competing events when one subtype was evaluated. Person-years (PY) were calculated from the date of participation in HUNT2 to the date when lung cancer was diagnosed, death occurred due to other reasons, the person emigrated out of Norway or follow-up ended (December 31, 2014), whichever occurred first. None of the variables showed evidence against the proportional hazards assumption.

In Model 1, the value of pack-years of active smoking was adjusted. In Model 2, age, sex, family history of cancer, education and economic difficulties were adjusted in addition to pack-years of active smoking. Model 3 adjusted for body mass index (BMI), physical activity, alcohol consumption and chronic bronchitis in addition to the variables included in Model 2. Lifestyle factors such as BMI, physical activity and alcohol consumption tend to cluster together, but their association with lung cancer is not yet confirmed. Chronic bronchitis might be either a confounder or in the causal pathway between passive smoking and lung cancer. Results from Model 2 were thus presented as the main findings. The potential effect modification by sex or active smoking status was evaluated by the Wald test. All statistical analyses

@ERSpublications

Childhood and adulthood exposure to passive smoking associated with increased risk of lung cancer http://ow.ly/7kXN30f6aZu

Cite this article as: Sun Y-Q, Chen Y, Langhammer A, et al. Passive smoking in relation to lung cancer incidence and histologic types in Norwegian adults: the HUNT study. Eur Respir J 2017; 50: 1700824 [https://doi.org/10.1183/13993003.00824-2017]. 
were performed using Stata/SE 13.1 software. The study was approved by the Regional Committee for Medical Research Ethics.

Among the 56772 subjects with a median follow-up time of 18.2 years, 692 subjects were diagnosed with lung cancer, 10601 subjects died owing to other reasons, and 228 subjects emigrated out of the country. All three passive smoking groups had significantly increased incidence of lung cancer overall (table 1), with the SHR being 1.50 for the passive smoking in childhood only group, 1.50 for the adulthood only group and 1.52 for the both periods group (Model 2). The incidence appeared to increase for both small cell lung cancer (SCLC) and nonsmall cell lung cancer (NSCLC) in all the three passive smoking groups, but the $95 \%$ confidence intervals were much wider for SCLC owing to the smaller number of cases. Regarding NSCLC, stronger associations were observed for squamous cell carcinoma (SHRs 2.65-3.05 across the passive smoking groups in Model 2) than for adenocarcinoma (SHRs 1.44-1.61). No statistically significant modification by sex or active smoking status was observed for either lung cancer overall or different histologic types.

In this prospective cohort study, we found that the three passive smoking groups (passive smoking in childhood only, in adulthood only, and in both periods) were associated with a $50-52 \%$ increased risk of lung cancer overall after adjustment for active smoking and other confounders. The risks appeared stronger for SCLC and squamous cell carcinoma but weaker for adenocarcinoma.

Both of the aforementioned meta-analysis articles, including mostly the case-control studies, demonstrated an approximate $30 \%$ increased risk of lung cancer associated with passive smoking in never-smokers $[1,2]$. The difference in the magnitude of the observed association in our study may be due to the difference in study design and possible residual confounding of active smoking. However, passive smoking in adulthood only and in both periods in never-smokers in our study was associated with a greater than $50 \%$ increased risk for lung cancer, although this figure was not statistically significant owing to the

TABLE 1 The association of passive smoking with lung cancer overall and histologic types in different models

\begin{tabular}{|c|c|c|c|c|c|c|c|c|}
\hline Passive smoking group & $\begin{array}{c}\text { Cases } \\
\mathrm{n}\end{array}$ & $\begin{array}{c}\text { Incidence rate } \\
100000 \text { person-years }\end{array}$ & \multicolumn{2}{|c|}{ Model 1} & \multicolumn{2}{|c|}{ Model 2} & \multicolumn{2}{|c|}{ Model 3} \\
\hline Lung cancer overall & $692^{\#}$ & & & & & & & \\
\hline Childhood only & 70 & 32.1 & 1.15 & $(0.79-1.66)$ & 1.50 & $(1.04-2.18)$ & 1.50 & $(1.04-2.18)$ \\
\hline Adulthood only & 176 & 118.0 & 1.70 & $(1.22-2.37)$ & 1.50 & $(1.08-2.08)$ & 1.50 & $(1.08-2.07)$ \\
\hline Both periods & 398 & 96.6 & 1.33 & $(0.97-1.83)$ & 1.52 & $(1.11-2.09)$ & 1.51 & $(1.10-2.08)$ \\
\hline Childhood only & 10 & 4.6 & 2.54 & (0.70-9.23) & 2.96 & (0.81-10.89) & 2.91 & $(0.79-10.74)$ \\
\hline Adulthood only & 24 & 16.1 & 3.03 & (0.90-10.24) & 2.39 & $(0.72-7.95)$ & 2.31 & $(0.69-7.72)$ \\
\hline Both periods & 52 & 12.6 & 2.24 & $(0.69-7.31)$ & 2.22 & $(0.67-7.35)$ & 2.17 & $(0.65-7.20)$ \\
\hline NSCLC & $421^{\pi}$ & & & & & & & \\
\hline Never & 22 & 12.5 & 1.00 & & 1.00 & & 1.00 & \\
\hline Childhood only & 46 & 21.1 & 1.65 & $(1.00-2.74)$ & 2.06 & $(1.23-3.43)$ & 2.06 & $(1.23-3.43)$ \\
\hline Childhood only & 22 & 10.1 & 1.32 & $(0.67-2.62)$ & 1.61 & $(0.81-3.19)$ & 1.58 & $(0.80-3.14)$ \\
\hline Adulthood only & 44 & 29.5 & 1.70 & $(0.91-3.17)$ & 1.44 & $(0.77-2.69)$ & 1.44 & $(0.77-2.68)$ \\
\hline Both periods & 110 & 26.7 & 1.49 & $(0.83-2.67)$ & 1.54 & $(0.86-2.76)$ & 1.50 & $(0.83-2.71)$ \\
\hline Squamous cell carcinoma & 136 & & & & & & & \\
\hline Never & 5 & 2.8 & 1.00 & & 1.00 & & 1.00 & \\
\hline Childhood only & 15 & 6.9 & 2.38 & $(0.86-6.56)$ & 3.05 & $(1.08-8.57)$ & 3.12 & $(1.12-8.71)$ \\
\hline Adulthood only & 32 & 21.5 & 2.74 & $(1.03-7.27)$ & 2.65 & $(1.00-7.02)$ & 2.69 & $(1.01-7.12)$ \\
\hline Both periods & 84 & 20.4 & 2.49 & $(0.97-6.38)$ & 2.99 & $(1.15-7.77)$ & 3.04 & $(1.18-7.85)$ \\
\hline
\end{tabular}

Model 1 was adjusted for pack-years of active smoking daily. Model 2 was adjusted for sex, age, family history of cancer, education and economic difficulties in addition to the variables included in Model 1. Model 3 was adjusted for body mass index, physical activity, alcohol consumption and chronic bronchitis at baseline in addition to the variables included in Model 2. SHR: subdistribution hazard ratio; SCLC: small cell lung cancer; NSCLC: nonsmall cell lung cancer. ${ }^{\#}$ : including 182 unknown types; " ${ }^{\text {: }}$ including 20 other types and 76 unknown NSCLC. 
smaller number of cases (data not shown). In our study no significant effect modification by sex was observed for either lung cancer overall or histologic types, which is in agreement with the meta-analysis of case-control studies [1]. This also confirms a previous prospective study suggesting that men and women are equally susceptible to tobacco carcinogens in the lung [9]. SCLC and squamous cell carcinoma are mainly located in the large bronchi [10], and it is reasonable to assume that the proximal sites in the respiratory tract are more intensely exposed to tobacco smoke particles than the peripheral sites [1].

There may be decades of delay between the initiation of smoking exposure and lung cancer incidence [11]. The association presented in the current study generally reflected the situation before the time that more restrictive measures on smoking in public places in the Norwegian Tobacco Act were enacted in the middle of the 1990s [12]. However, passive smoking is still prevalent in many other countries [13-15]. Thus, it is important to continue disseminating the message about the potential hazards of passive smoking and promoting smoking cessation to reduce lung cancer incidence to the largest extent possible.

This study has several potential limitations. First, misclassification due to inaccuracies in the self-reporting of passive smoking is a concern. Although exposure information was collected before the diagnosis of lung cancer and differential misclassification was less likely, association estimates should be interpreted with caution. Second, our passive smoking status reflected mainly at-home exposure, but not the total exposure. Third, one possible explanation for the non-observed cumulative effect of passive smoking in both periods may be the lack of information on the quantitative characteristic of exposure to passive smoking, such as duration and amount. Nevertheless, our study is one of the first prospective cohort studies to investigate the association between passive smoking with lung cancer overall and major histologic types in both men and women.

In conclusion, in this long-term prospective study we found that childhood and adulthood reported exposure to passive smoking was associated with an increased risk of lung cancer in both men and women. The risks appeared stronger for SCLC and squamous cell carcinoma but weaker for adenocarcinoma.

Yi-Qian Sun ${ }^{1}$, Yue Chen ${ }^{2}$, Arnulf Langhammer ${ }^{3}$, Frank Skorpen ${ }^{1}$, Chunsen $\mathrm{Wu}^{4,5}$ and Xiao-Mei Mai ${ }^{3}$

${ }^{1}$ Dept of Laboratory Medicine, Children's and Women's Health (LBK), NTNU, Norwegian University of Science and Technology, Trondheim, Norway. ${ }^{2}$ School of Epidemiology, Public Health and Preventive Medicine, Faculty of Medicine, University of Ottawa, Ottawa, ON, Canada. ${ }^{3}$ Dept of Public Health and Nursing, NTNU, Norwegian University of Science and Technology, Trondheim, Norway. ${ }^{4}$ Institute of Clinical Research, University of Southern Denmark, Odense, Denmark. ${ }^{5}$ Odense University Hospital, Odense, Denmark.

Correspondence: Yi-Qian Sun, Dept of Laboratory Medicine, Children's and Women's Health (LBK), NTNU, Norwegian University of Science and Technology, Trondheim, Norway. E-mail: yi-qian.sun@ntnu.no

Received: March 302017 | Accepted after revision: July 142017

Support statement: Y-Q. Sun's research position is supported by funding from The Norwegian Cancer Society (project ID 5769155-2015) and The Research Council of Norway "Gaveforsterkning". The study used data from the Cancer Registry of Norway. The interpretation and reporting of these data are the sole responsibility of the authors, and no endorsement by the Cancer Registry of Norway is intended nor should be inferred. Funding information for this article has been deposited with the Crossref Funder Registry.

Conflict of interest: Disclosures can be found alongside this article at erj.ersjournals.com

Acknowledgements: The Nord-Trøndelag Health Survey (HUNT) is a collaboration between the HUNT Research Centre (Faculty of Medicine and Health Sciences, NTNU, Norwegian University of Science and Technology), the Nord-Trøndelag County Council and the Norwegian Institute of Public Health.

Author contributions: YQS, AL, YC and XMM contributed to the study design. XMM and AL contributed to data collection. CW contributed to statistical analyses. YQS conducted statistical analyses, interpreted results and wrote the initial draft of the manuscript. AL, FS, CW, YC and XMM participated in the data interpretation and helped to write the final draft of the manuscript.

\section{References}

1 Kim CH, Lee YC, Hung RJ, et al. Exposure to secondhand tobacco smoke and lung cancer by histological type: a pooled analysis of the International Lung Cancer Consortium (ILCCO). Int J Cancer 2014; 135: 1918-1930.

2 Taylor R, Najafi F, Dobson A. Meta-analysis of studies of passive smoking and lung cancer: effects of study type and continent. Int J Epidemiol 2007; 36: 1048-1059.

3 Jee SH, Ohrr H, Kim IS. Effects of husbands' smoking on the incidence of lung cancer in Korean women. Int $J$ Epidemiol 1999; 28: 824-828.

4 Kurahashi N, Inoue M, Liu Y, et al. Passive smoking and lung cancer in Japanese non-smoking women: a prospective study. Int J Cancer 2008; 122: 653-657.

5 Wang A, Kubo J, Luo J, et al. Active and passive smoking in relation to lung cancer incidence in the Women's Health Initiative Observational Study prospective cohort. Ann Oncol 2015; 26: 221-230. 
6 Cancer in Norway 2014: cancer incidence, mortality, survival and prevalence in Norway. Oslo, Cancer Registry of Norway, 2015.

7 International Classification of Diseases for Oncology (ICD-O-3). 3rd Edn. Geneva, World Health Organization, 2013.

8 Andersen PK, Geskus RB, de Witte T, et al. Competing risks in epidemiology: possibilities and pitfalls. Int $J$ Epidemiol 2012; 41: 861-870.

9 Freedman ND, Leitzmann MF, Hollenbeck AR, et al. Cigarette smoking and subsequent risk of lung cancer in men and women: analysis of a prospective cohort study. Lancet Oncol 2008; 9: 649-656.

10 Morabia A, Wynder EL. Cigarette smoking and lung cancer cell types. Cancer 1991; 68: 2074-2078.

11 Thun MJ, Henley SJ, Calle EE. Tobacco use and cancer: an epidemiologic perspective for geneticists. Oncogene 2002; $21: 7307-7325$

12 Milestones in Norwegian tobacco control. https://helsedirektoratet.no/english/tobacco-control\#milestones-innorwegian-tobacco-control Date last accessed: August 7, 2017. Date last updated: July 3, 2017.

13 Britton J, Bogdanovica I. Tobacco control efforts in Europe. Lancet 2013; 381: 1588-1595.

14 Muttarak R, Steiber N, Gallus S. Smoking ban in Austria: a long overdue step but still a lot to be done. Lancet 2015; 385: 941-942.

15 Yang G, Wang Y, Wu Y, et al. The road to effective tobacco control in China. Lancet 2015; 385: 1019-1028. 